\title{
EMPIRICAL DATA ON AGE AND POSITION \\ IN THE HERTZSPRUNG-RUSSELL DIAGRAM \\ FOR O AND B-TYPE STARS
}

A. BLAAUW

Kapteyn Laboratory, Groningen

For satisfactory tests of the theory of the evolution of the massive stars, accurate data are required on their positions in the HR diagram together with the corresponding ages. The following remarks deal with two pieces of information relevant to this problem.

H I GH - VELOCITY O A N D - T Y P E S TAR S

Attention has been drawn to the high-velocity $\mathrm{O}$ and B-type stars in previous papers by the author, I956 and $1958[\mathrm{I}, 2]$. For some of these objects both the luminosity and the "expansion age" can be determined. Such data are summarized in Table $I$; the ages and visual absolute magnitudes are in the 6th and 7 th column, respectively. The associations from which the stars originated are indicated in the 5th column. For all of them except for ro Lac the distance from this origin, as well as the distance from the sun and the speed with respect to the association, are known well enough to allow a determination of the time elapsed since the star left the origin (the "expansion age") and its visual absolute magnitude. The distance moduli used in the computations are as follows: II Per, 7.4; I Cep, 9.3; I Ori, 8.3; I Sco, 6.3; I Lac, 8.6. The $\mathrm{O}_{9}$ star Io Lac has been added to the list although this is not a high-velocity object, but its age may be assumed to be approximately that of the association I Lac as found by Blaauw and Morgan [3].

The positions in the HR diagram are shown in Fig. I and the expansion ages (in millions of years) are indicated near each dot. The drawn line in the lower right part represents the "zero-age" main sequence according to Johnson [4]; (age in that nomenclature being counted

Table I

Early Type Stars with Known Expansion Age and Luminosity

\begin{tabular}{|c|c|c|c|c|c|c|}
\hline Name & $\begin{array}{l}\text { HD } \\
\text { No. }\end{array}$ & $\begin{array}{c}\text { MK } \\
\text { Spectral } \\
\text { Type }\end{array}$ & $\begin{array}{l}\text { Space } \\
\text { Velocity } \\
\text { (km/sec) }\end{array}$ & $\begin{array}{l}\text { Associated } \\
\text { with }\end{array}$ & $\begin{array}{c}\text { Expansion } \\
\text { Age } \\
\text { (106 } \text { yrs }^{6}\end{array}$ & $\mathbf{M}_{\mathbf{v}}$ \\
\hline $\begin{array}{l}\xi \text { Per } \\
68 \text { Cyg } \\
\text { AE Aur } \\
\zeta \text { Oph } \\
\mu \text { Col } \\
53 \text { Ari } \\
\text { Io Lac }\end{array}$ & $\begin{array}{r}24912 \\
203064 \\
34078 \\
149757 \\
38666 \\
19374 \\
214680\end{array}$ & $\begin{array}{l}\mathrm{O} 7 \\
\text { O8 } \\
\text { O9.5 V } \\
\text { O9.5 } \\
\text { Bo V } \\
\text { B2 V } \\
\text { O9 }\end{array}$ & $\begin{array}{r}49 \\
45 \\
\text { I } 17 \\
39 \\
125 \\
66\end{array}$ & $\begin{array}{l}\text { II Per } \\
\text { I Cep? } \\
\text { I Ori } \\
\text { I Sco } \\
\text { I Ori } \\
\text { I Ori } \\
\text { I Lac }\end{array}$ & $\begin{array}{l}2.0 \\
5.1: \\
2.7 \\
1.1 \\
2.4 \\
4.9 \\
4.2\end{array}$ & $\begin{array}{l}-4.8 \\
-5.1: \\
-4.0 \\
-4.3 \\
-3.7 \\
-2.6 \\
-4.1\end{array}$ \\
\hline
\end{tabular}


from the time a star has arrived on the main sequence). The dashed line represents its continuation towards earlier spectral types suggested by the data of Table I. We find :

(A) No stars younger than one million years appear among the objects studied, and these are located close to the main sequence. Apparently the stars make their appearance in this region of the HR diagram rather suddenly. Objects of still more recent formation than those

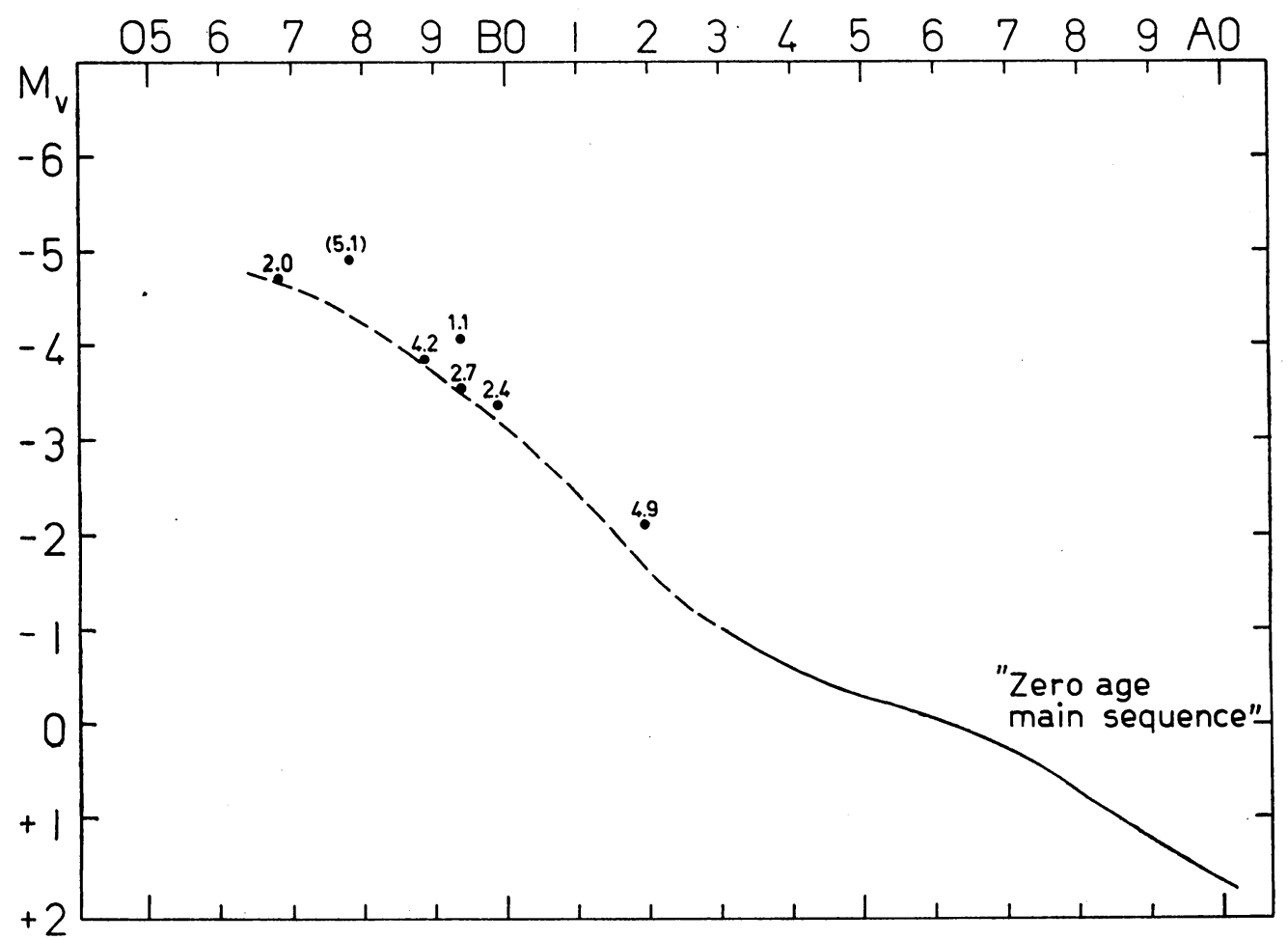

Figure I. Positions in the HR diagram for the stars of Table I. The figures written next to the dots indicate the "expansion ages" in millions of yars.

listed and with similar speeds will probably also exist. Such stars should be found closer to the associations from which they originated than the objects of Table I, and therefore will not have escaped detection if they are of a brightness comparable to that of the objects listed. A rough estimate based on the scarce available data, which hardly justify statistical considerations, indicates that stars on their way to the main sequence must spend less than 200000 years on the section of their tracks in the region of the diagram brighter than absolute magnitude -2 and at types earlier than $\mathrm{B} 3$.

(B) So far, no stars with expansion ages exceeding 5 million years have been found among the high-velocity $\mathrm{O}$ stars. But this may well be due to observational selection. These objects will in general have moved so far from their origin that present observations do not allow them to be associated with certainty with the known associations.

THE SCORPIO-CENTAURUS ASSOCIA T I O N

The short distance of this association from the sun (I8o psc) and its large dimensions, allow an accurate determination of individual distances of its members. This has most recently 


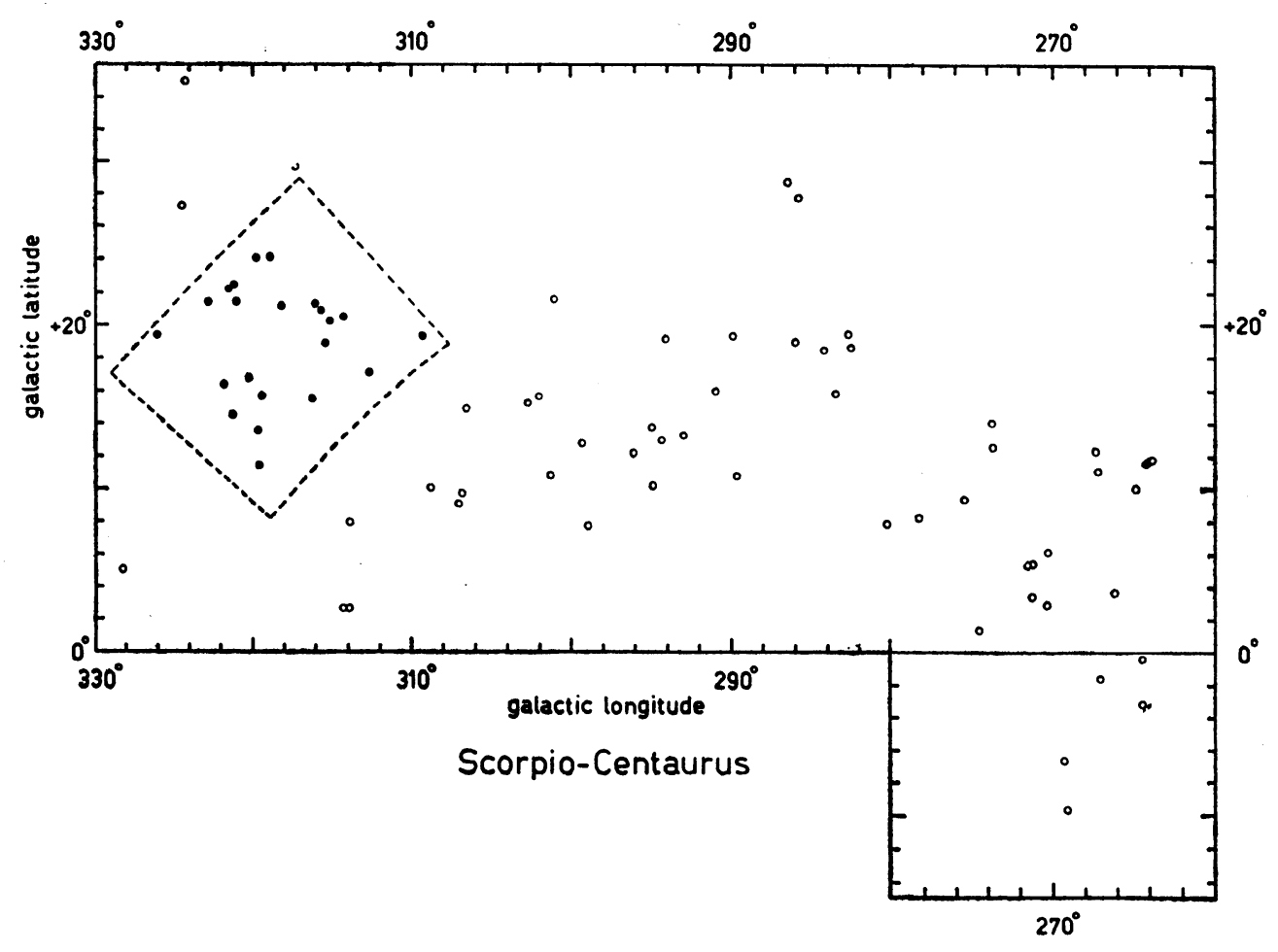

FIgURE 2. Apparent distribution on the sky for the brighter members of the Scorpio-Centaurus association according to galactic coordinates. The dashed square indicates the region strongly associated with interstellar matter.

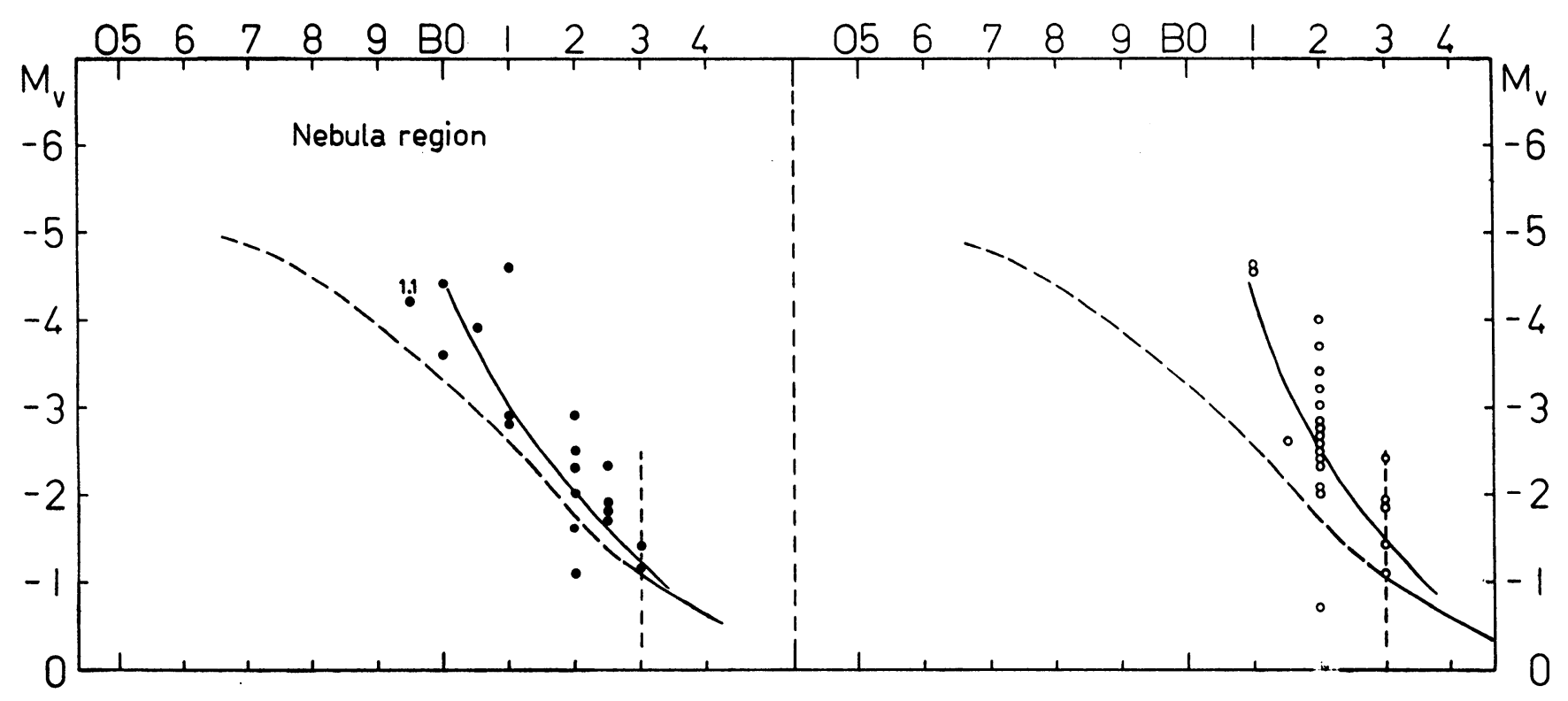

Scorpio-Centaurus

$$
\left(20 \times 10^{6} \mathrm{yrs}\right)
$$

FIGURE 3. HR diagram for the Scorpio-Centaurus association, subdivided into two parts. The left-hand diagram represents the stars in the nebula region, corresponding with the dashed square in Figure $2 ;$ the right-hand diagram represents the remaining part of the association. The main sequence curve is the same as that drawn in Figure $I$. 
been done by Bertiau [5]. Of particular interest is the fact, that the association, like many other associations, can be subdivided into a part involved in nebulosity, in which the population is relatively dense, and a remaining part of lower density, free of interstellar matter. Figure 2 shows the apparent distribution of the members in galactic coordinates for longitudes above $260^{\circ}$. The northern, most densely populated, part is heavily associated with nebulosities; it includes the bright nebulae and dark lanes around $\rho$ Ophiuchi. This part is approximately contained in the dashed square. The stars inside and outside this square are represented by dots and circles, respectively.

Figure 3 shows the HR diagram for these two subdivisions; the same symbols were used as for the two divisions of Fig. 2 and the main sequence curve has been copied from Fig. I. The observed arrays suggest that the stars in the loose, clear region (right hand diagram) have further evolved from the main sequence than those of the nebula region. For instance, the average luminosity of the B2 stars is about 0.5 magnitude fainter in the nebula region than in the remaining part. The age difference is in the sense one would expect from the different degrees of association with nebulosity; the younger part being (still) more immersed in it than the older.

Bertiau's analysis has led to an age estimate of about 20 million years. This is based on the interpretation of the $\mathrm{K}$ term as due to general expansional motion. It should apply to the oldest part rather than to the association as a whole. Accordingly an expansion age of 20 million years should be assigned to the mean locus of the circles in the right hand part of Fig. 3.

[1] Blaauw, A., Publ. Astr. Soc. Pac., 68, 495, 1956.

[2] Blaauw A., Semaine d'Étude sur le Problème des Populations Stellaires, Pont. Acad. Scient. Scripta Varia, 16, 105, 1958; Specola Astr. Vaticana Ric. Astr. 5 (ed. D. J. K. O’Connell S. J.).

[3] Blaauw, A. and Morgan, W. W., Ap. J., 117, 256, 1953.

[4] Johnson, H. L., $A p . J ., 126,121,1957$.

[5] Bertiau, F. C., Ap. J., 128, 533, 1958. 